\title{
Serial Subtractive Deconvolution Algorithms for Time-Domain Ultra Wide Band In-Vehicle Channel Sounding
}

\author{
Aniruddha Chandra ${ }^{1}$, Jiří Blumenstein ${ }^{1}$, Tomáš Mikulášek ${ }^{1}$, Josef Vychodil ${ }^{1}$, Roman Maršálek ${ }^{1}$, \\ Aleš Prokeš ${ }^{1}$, Thomas Zemen ${ }^{2,3}$, and Christoph F. Mecklenbräuker ${ }^{4}$ \\ ${ }^{1}$ Department of Radio Electronics, Brno University of Technology, 61600 Brno, Czech Republic. \\ 2 AIT Austrian Institute of Technology GmbH, 1220 Vienna, Austria. \\ ${ }^{3}$ Telecommunications Research Center Vienna (FTW), 1220 Vienna, Austria. \\ ${ }^{4}$ Institute of Telecommunications, Vienna University of Technology, 1040 Vienna, Austria.
}

\begin{abstract}
Ultra wide band (UWB) communication is expected to play a key role in next generation broadband intra vehicle wireless applications. The car compartment differs significantly from other well studied indoor or outdoor environments. Hence, channel sounding experiments are crucial for gaining a thorough knowledge of the UWB signal propagation characteristics in such a medium. Time domain channel sounding campaigns often employ some sort of deconvolution during measurement post processing as the measured signal in these experiments is the convolution of the channel response and the probing pulse which violates the Nyquist criterion. In this paper, a comparison of two variants of time-domain serial subtractive deconvolution algorithm, popularly known as CLEAN, is presented. Appropriate statistical metrics for assessing the relative merit of a deconvolution technique are identified in the context of intra vehicle UWB transmission, and the better algorithm is selected based on its performance over a standard IEEE channel simulation testbed. The chosen method is then applied to extract power delay profile and delay parameters from an empirical time domain sounding experiment performed inside a passenger car. The effects of passenger occupancy, transmitter receiver separation, and absence of direct transmission path are studied.
\end{abstract}

Keywords: Deconvolution, CLEAN, ultra wide band, IEEE 802.15.3, intra-vehicle communication, channel sounding.

\section{Introduction}

\subsection{Motivation}

Intra-vehicular communication, in the conventional sense, refers to transmission of signals between electronic control units (ECUs) and sensors/ actuators over a wired common control area network (CAN) bus $[1,2,3]$. The degree of sophistication for automotive vehicles regarding safety, driver assistance, and passenger comfort has escalated multifold over the years increasing the overall wiring harness of the in-vehicle electrical network. With the enhanced penetration of Internet and satellite networks in all spheres of modern life, demand for yet another kind of intra-vehicular communication is in rise. According to a survey by Juniper Research, 20\% cars in the US and Western Europe will be able to access Internet apps by 2017. Thus, navigational and recreational infotainment networks including live audio/ video streaming facilities are expected to be an integral part of every car in near future [4]. As far as the wiring harness is considered, this will surely add up to the already existing cable bundle of several kilometers [5] that run inside a car posing severe design and manufacturing challenges to the automobile industry. 
Replacing intra-vehicular wired cables and connectors with wireless links seems to be a promising alternative and offers the following advantages. First, reducing the vehicle weight by $40 \mathrm{~kg}$, typical wiring harness for present day mid-sized cars [6], would increase fuel efficiency by 2 percent. The value is not very small by automotive engineering standards. On the other hand, the ecological impact gained through reduction of greenhouse gas emission is invaluable. Second, eliminating the cables will reduce cost and labour in design, manufacturing, and installation processes. Third, maintenance becomes less likely and much easier without the long running cables cramped in a closed space [7]. However, one should not forget the inherent limitations of wireless communication, especially the random nature of the channel which makes the wireless links unsuitable for safety-critical communications. Also, wireless power transfer is still in its infancy and current endeavours are directed towards replacement of data cables only. Power cables are going to stay, and might even increase as every new wireless in-vehicle device requires connection to the electrical power source [1]. Nevertheless, the incentives of wireless harness are attracting huge research fundings, and the quest for replacing cable bundles has already been extended to aircrafts [8] and spacecrafts [9], where both the rewards and stakes are definitely much higher.

Initial candidates for providing wireless access in intra-car applications included narrowband standards such as radio frequency identification (RFID), Bluetooth, wireless 1394 [10], and IEEE 802.11 (WiFi) among others. A recent ruling by the European Commission now allows implementation of short range wideband wireless standards inside cars and trains [11], and gradually the focus of intra-car wireless research is now being shifted to tests and measurements in the ultra wide band (UWB) $[12,13,14,15,16]$ and in millimeter wave (mmW) bands $[17,18,19,20]$.

UWB promises very high-data-rate communication, in the order of several hundred Mbps, across distances up to a couple of meters. UWB also provides a reliable means of communication even in environments traditionally considered very hostile to radio by mitigating large-scale (shadowing) as well as small-scale (multipath) fading effects. Furthermore, the low power spectral density of UWB signals ensures minimum interference to nearby cars or fixed wireless infrastructures. UWB propagation characteristics has been already well studied [21] in the context of various indoor and outdoor environments. However, a passenger car compartment with metal body and plastic / foam interiors, is a different environment altogether. Confined structures and human presence affects UWB propagation [22] significantly necessitating channel sounding experiments for gaining a thorough knowledge of the propagation characteristics.

In general, there are two possible methods for wideband channel sounding [23, 24]. The first is sounding in frequency-domain with a vector network analyzer (VNA), where the channel is swept at different frequencies. The transfer function parameter $(s)$ provides an estimate of the channel transfer function, $\hat{H}(f)$, which

may be used to obtain the impulse response estimate, $\hat{h}(t)=\mathcal{F}^{-1} \hat{H}(f)$, through inverse fast Fourier transform (IFFT). The second method, time-domain sounding, is more direct where short pulses are sent over the channel and the channel's impulse response convolved with the pulse shape is recorded by an oscilloscope. A desired feature of any wideband time domain sounding is its super-resolution capability [25], i.e. having the potential to distinguish between multi-path components that are separated by a time duration lesser than the channel sounding pulse width. The goal can be attained by time-domain subtractive deconvolution algorithms (e.g. CLEAN).

\subsection{Literature Survey}

Different versions of CLEAN had been successfully implemented in geotechnical seismic analysis, spectroscopy in radio astronomy, and biomedical image processing for last few decades [26]. Multi-template case specific CLEAN algorithms for UWB were also reported in [27] and [28]. However, intra-vehicular measurement literature is not particularly rich in this regard as most of the channel sounding were performed 
in frequency domain [29, 30, 31].

Time domain experimental papers often lacked detailed discussion on the implementation of algorithm. The only exception is [16], where a sub-optimal version of CLEAN is presented. In this paper we show that the modified version of the algorithm can perform much better in intra-vehicular environments. Part of these results was published in a conference paper [32]. The experimental domain of the current paper is also entirely different from [16]. While the authors in [16] characterized wireless channels in the engine compartment and under the chasis, with an objective of possible sensor network implementation in vehicles, we carried our measurements in the passenger compartment, and the target application is localization of people and devices inside the car. Another important goal of this paper is to study the effect of passenger occupancy on the channel parameters.

It may be noted here that a frequency domain successive subtraction algorithm for UWB was presented in [33]. This algorithm has reduced complexity compared to expectation-maximization (EM) [34] or spacealternating generalized EM (SAGE) [35] algorithms.

\subsection{Contributions of the Paper}

Although we provide an exhaustive description of the UWB measurement campaign which is a part of our GACR project [36] aimed at intra-vehicular channel modelling in UWB, mmW, and infra-red (IR) domains, our main contribution lies in the choice of deconvolution and its validation. The selection of a proper deconvolution algorithm is an important step in any time-domain channel sounding experiment. This is particularly important for UWB channel sounding where pulse shape at the transmitter and receiver can not be chosen according to the Nyquist theorem. Usual channel sounders use a root raised cosine pulse such that the total impulse response gives a Nyquist pulse.

Moreover, study of CLEAN is beneficial in another sense because the algorithm provides discrete impulse response in time domain and helps in locating the first peak resulting from the direct path (or most dominant multipath) which is crucial in localization applications, the ultimate goal of the GACR project.

Specifically, our contributions are:

- We present discrete-time versions of CLEAN algorithms that are readily executable through software packages.

- We identify appropriate statistical metrics for comparing deconvolution methods and validate our choice through standard UWB testbed simulation.

- We obtain channel impulse response for various transmitter receiver positions in the passenger compartment of a car through deconvolution operation.

- We calculate power delay profile, mean excess delay, and RMS delay spread from the channel impulse responses.

- We study the effects of passenger occupancy, transmitter receiver separation, and obstruction of line of sight path on intra-vehicle wireless channel parameters.

\subsection{Organization of the Paper}

The structure of this paper is as follows. Section 2 describes the overall measurement campaign in detail. In particular, the choice of the sounding pulse, the measurement environment, the antenna placement, and the measurement setup are discussed. Section 3 provides two working versions of the CLEAN algorithm and 
explains the intricacies of comparison between them. The section also includes the post-processing of the measured data. The calculation of power delay profile and multipath delay parameters that can be extracted from the measured data as well as the effects of distance, passenger occupancy, and line-of-sight condition are presented in Section 4. Finally, Section 5 concludes the paper.

\section{Measurement Campaign}

\subsection{Sounding Pulse}

Due to the availability of cheap and uncomplicated hardware, the UWB systems that were designed and implemented so far are mostly of the type impulse radio (IR-UWB) transmitting some form of Gaussian pulses. A basic Gaussian pulse, popularly known as Gaussian monocycle, is defined as

$$
p(t)=\sqrt{\frac{1}{t_{d}} \sqrt{\frac{2}{\pi}}} \exp \left[-\left(\frac{t}{t_{d}}\right)^{2}\right]
$$

having unit energy and an effective pulse duration of $2 t_{d}$.

The basic pulse, however, possesses a non-zero DC-offset and cannot be efficiently radiated through antennas of realizable dimensions. The widely adopted version is a Gaussian doublet, the second derivative $\left(p_{2}(t)=\partial^{2} p(t) / \partial^{2} t\right)$ of the Gaussian pulse

$$
p_{2}(t)=-2 \sqrt{\frac{1}{3 t_{d}} \sqrt{\frac{2}{\pi}}}\left(1-\frac{2 t^{2}}{t_{d}^{2}}\right) \exp \left[-\left(\frac{t}{t_{d}}\right)^{2}\right]
$$

which is obtained at the output of the transmitting antenna if the antenna is fed with a current pulse proportional to the first derivative, $p_{1}(t)=\partial p(t) / \partial t$, owing to the derivative nature of the antenna [21]. Noting that the Fourier transform (FT) of the basic pulse is

$$
P(f)=\mathcal{F}\{p(t)\}=\sqrt{t_{d} \sqrt{2 \pi}} \exp \left[-\left(\pi t_{d} f\right)^{2}\right]
$$

and utilizing the derivative property of the FT, it is easy to obtain the amplitude spectra of a Gaussian doublet from (3)

$$
P_{2}(f)=-2 \sqrt{\left(t_{d} / 3\right) \sqrt{2 \pi}}(j 2 \pi f)^{2} \exp \left[-\left(\pi t_{d} f\right)^{2}\right]
$$

where $j=\sqrt{-1}$ denotes the complex square root of unity. When plotted (see Fig. 1, right-top, black curve), the spectra shows significant low frequency $(<3 \mathrm{GHz})$ components in spite of having a zero DCoffset. The implications can be better understood through a comparison of the energy spectral density (ESD) with the effective isotropic radiated power (EIRP) emission mask standardized by Federal Communications Commission (FCC) [37], as shown in Fig. 1 [right-bottom]. The figure clearly reveals that for the given pulse parameters, the Gaussian doublet (black curve) violates the regulations, and in order to keep the EIRP in limit, the signal strength needs to be reduced causing a drop in the SNR of about $20 \mathrm{~dB}$. The problem is not typical for Gaussian shaped pulses, and exists for other baseband pulses too, say, for example, a raised cosine pulse that obeys Nyquist criterion.

The frequency spectra may be shifted away further from the origin through a pseudo-carrier modulation with the help of a carrier having frequency not much higher than the bandwidth of the Gaussian pulse [38]. 

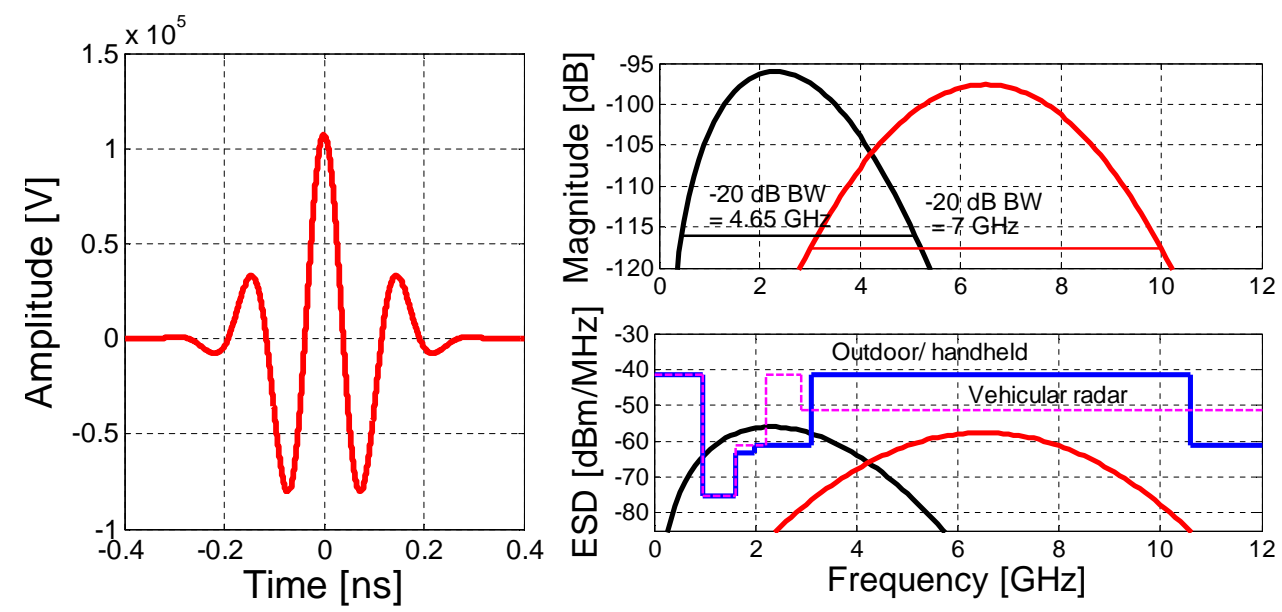

Figure 1: Input pulse $x(t)$ [left], it's amplitude spectra $X(f)_{+}$[right-top], and compliance with FCC UWB emission masks [right-bottom]. The black curves depict the frequency-domain characteristics for the Gaussian 2nd derivative with equivalent parameters (unit energy and same $t_{d}$ ).

This fact prompted us to use a sinusoidally modulated Gaussian pulse for our intra vehicular UWB channel sounding campaign. The unit energy sounding pulse (Fig. 1, left) used for the field measurements is given by

$$
x(t)=\sqrt{\frac{2}{t_{d}} \sqrt{\frac{2}{\pi}}} \exp \left[-\left(\frac{t}{t_{d}}\right)^{2}\right] \cos \left(2 \pi f_{c} t+\phi\right)
$$

having an initial phase of $\phi=0.6 \pi$ and a time scaling factor of $2 t_{d}=0.276 \mathrm{~ns}$. The carrier frequency, $f_{c}=6.5 \mathrm{GHz}$, was set at the middle of the FCC approved band, i.e. from $3 \mathrm{GHz}$ to $10 \mathrm{GHz}$. Applying the frequency shifting property of the FT, the corresponding spectra for positive frequencies (red curve) may be derived from (3)

$$
X(f)_{+}=\sqrt{\frac{2 t_{d}}{\pi}} \exp \left[-\left\{\pi t_{d}\left(f-f_{c}\right)\right\}^{2}\right]
$$

which is plotted alongside (4), the spectra of the Gaussian doublet (black curve), in Fig. 1 [right]. It is evident that the sounding pulse has a better compliance with the outdoor/ handheld and vehicular radar emission masks and holds stronger potential to be employed in future in-vehicle UWB communication systems. Further, when the $-20 \mathrm{~dB}$ bandwidths of the two pulse shapes are compared, we find that while the Gaussian doublet has a bandwidth of $4.65 \mathrm{GHz}$, the Gaussian sine pulse offers a $-20 \mathrm{~dB}$ bandwidth of $7 \mathrm{GHz}$ (bandwidth is almost 1.5 times) which results in a much flatter spectrum as desired by FCC and other regulating bodies.

\subsection{Description of the Environment}

The vehicle under study is a right-hand drive, regular four-door sedan Škoda Octavia III (model 1.8 TSI Combi) with dimensions $4.659 \mathrm{~m}$ (length) $\times 1.814 \mathrm{~m}$ (width) $\times 1.462 \mathrm{~m}$ (height), which was parked six storeys beneath ground level in the multi-floored underground garage of the Faculty of Electrical Engineering (FEKT), Brno University of Technology (VUT). Reinforced concrete walls and floors of the garage 
provided us with an environment that was free from any narrowband interference (e.g. WiFi, Cellular). Also, there were no other cars parked in close vicinity.
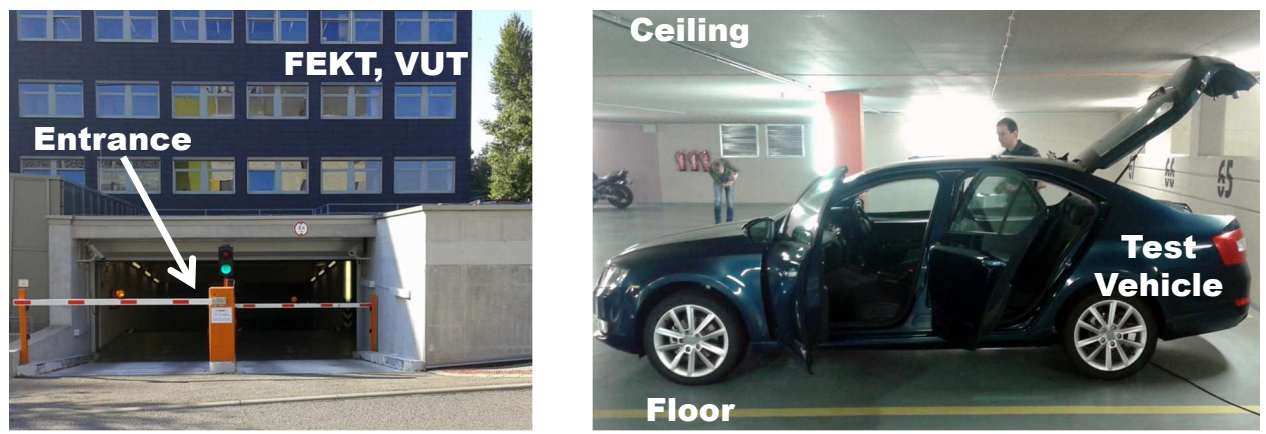

Figure 2: [left] Underground garage where the measurements were conducted. [right] Test vehicle and its surroundings.

Fig. 2 depicts the underground garage and the position of the vehicle in the parking lot. While recording the data, all the test equipments except the transmitter and receiver antennas were kept outside the car compartment. Further, all the doors and windows of the vehicle were closed except a small opening in the driver window allowing to pass the cables which connect antennas with measurement devices.

\subsection{Antenna Placement}

As shown in Fig. 3 [left], a total of 52 different transmitter (Tx) and receiver (Rx) antenna positions were tested with Tx-Rx separations ranging from $0.56 \mathrm{~m}$ to $1.9 \mathrm{~m}$. Distances between antennas were recorded for each setting with a flexible measuring tape. In addition, we also examined the effect of passenger occupancy by keeping some of these Tx-Rx setting fixed, and varying the number of passengers from zero to three. It may be noted that although the car can accommodate four persons, we could vary the passenger count (including the driver) only up to three, as one of the places was always occupied by the receiver antenna and its attachments.

The receiver antenna had been placed on the driver's seat (D) and on the seat behind the driver (RPR, $\mathrm{P}_{3}$ ) to imitate a hand-held mobile wireless device that belongs to either the driver or to a passenger. A plastic photographic tripod (JOBY GorillaPod) was used to maintain proper height (hand to lap separation) of the Rx antenna as well as to keep the inverted cone base of the antenna in horizontal position, while placing it on the seat cushion.

A wider variety of locations were set for the transmitter antenna spanning all over the car, including the left and right side of the dashboard $\left(\mathrm{L}_{1}, \mathrm{R}_{1}\right)$ and windshield $\left(\mathrm{L}_{2}, \mathrm{R}_{2}\right)$, the rear part of the ceiling $\left(\mathrm{P}_{1,2}\right)$, the luggage space $\left(\mathrm{P}_{4}\right)$, and the engine compartment $\left(\mathrm{P}_{5}\right)$. Determination of the Tx antenna positions were governed by two parallel objectives. First, the positions should resemble possible installation site for future in-vehicle wireless systems. For example, in modern cars position $\mathrm{P}_{1,2}$ may serve as a wireless docking station because this place usually contains some wiring and antenna for audio system or global positioning system (GPS). The second goal is to realize both line-of-sight (LoS) and non-line-of-sight (nLoS) scenarios. When the receiver was placed at driver's (D) seat, the measurement sets such as $R_{2}-D, L_{2}$-D etc. represented LoS wireless links. On the other hand, putting the receiver antenna in rear passenger seat on the right (RPR) resulted in $n L o S$ transmission $\left(\mathrm{R}_{2}-\mathrm{RPR}, \mathrm{L}_{2}-\mathrm{RPR}\right)$, due to the blocking by the backrest of the seats [39].

A pair of vertically polarized wideband monopole conical antennas (photos in Fig. 3) were used for 


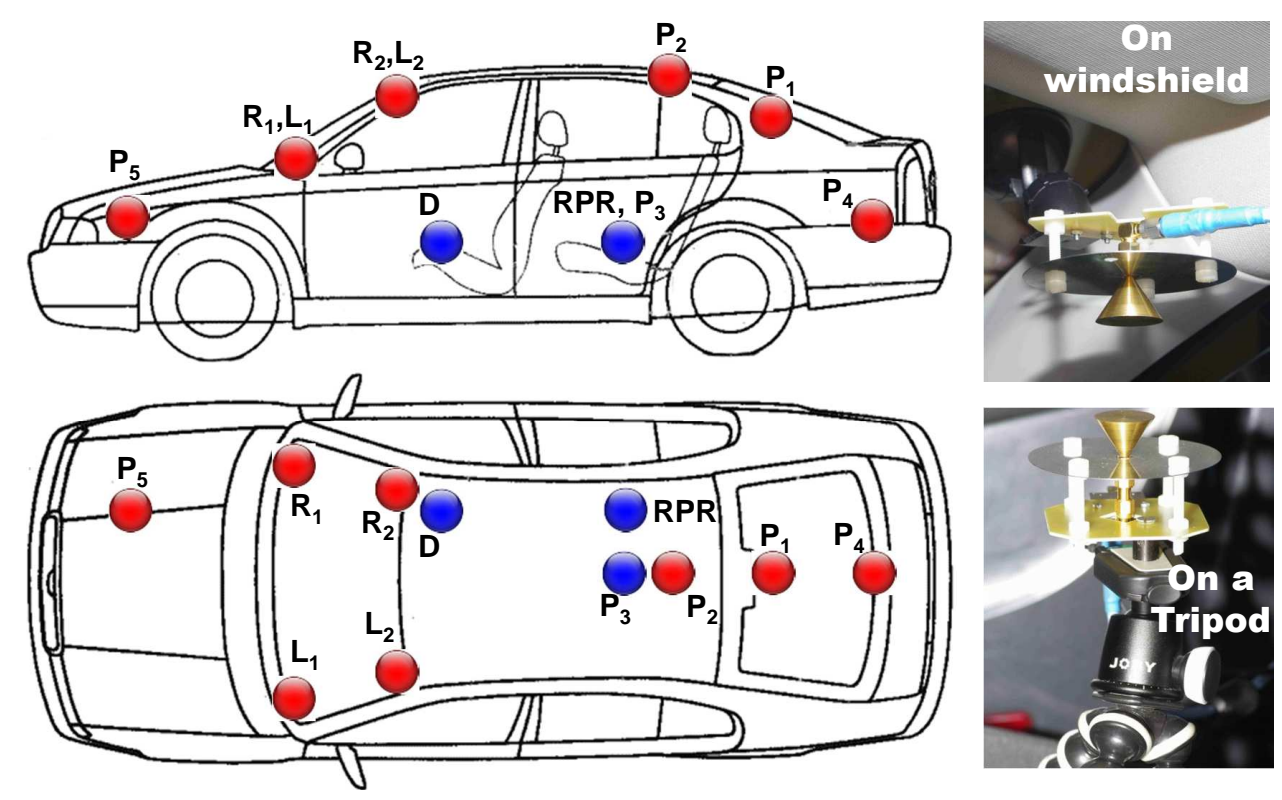

Figure 3: [left] Side-view and top-view schematic of antenna positions, RED: Tx antennas, BLUE: Rx antennas. [legends] D: driver, RPR: rear passenger on right, L/R: left and right windshield (subscripts 1 and 2 denote bottom and top), $\mathrm{P}_{1}-\mathrm{P}_{5}$ : position 1 to position 5 of the $\mathrm{Tx} / \mathrm{Rx}$ antenna. [right] Real world antenna placement close-ups: on right windshield (top), and on tripod (bottom).

both transmission and reception. The monopole conical antennas are omnidirectional, has a low radar crosssection (RCS), and provide a low voltage standing wave ratio (VSWR) [40]. Fig. 4 describes the simulated radiation pattern of the designed UWB antennas. It can be observed that the azimuth plane pattern is circular and omnidirectional which is invariant within the desired frequency band $(3-10 \mathrm{GHz})$.

\subsection{Measurement Setup}

The schematic diagram of the setup for UWB time domain channel sounding and an actual photograph of the field measurement device assembly are shown in Fig. 5.

The channel sounding pulse mentioned in (5) was generated at a sampling rate of $f_{s}=50 \mathrm{Gs} / \mathrm{s}$ through the Tektronix AWG70002A arbitrary waveform generator (AWG); after importing the corresponding waveform file from MATLAB. Next, the signal was amplified through a high-power amplifier (HPA) before feeding the signal to the transmitter antenna. We used Wenteq broadband power amplifier ABP1200-01-1825 which provided a gain of around $19 \mathrm{~dB}$.

The receiving portion of the measurement system consisted of an identical receiving antenna, a lownoise amplifier (LNA), and a digital sampling oscilloscope (DSO). For LNA, Wenteq ABL1200-08-3220 was used that had a small signal gain of $32 \mathrm{~dB}$ and a noise figure of $2 \mathrm{~dB}$. Finally, the received waveform was stored with the help of the mixed signal oscilloscope Tektronix DPO72004C.

Interconnections among devices and synchronization between AWG and DSO were realized through flexible coaxial cables. The total cable loss was about $5 \mathrm{~dB}$ in total. A programmable DC power supply Voltcraft PPS-11360 powered the HPA and the LNA. 


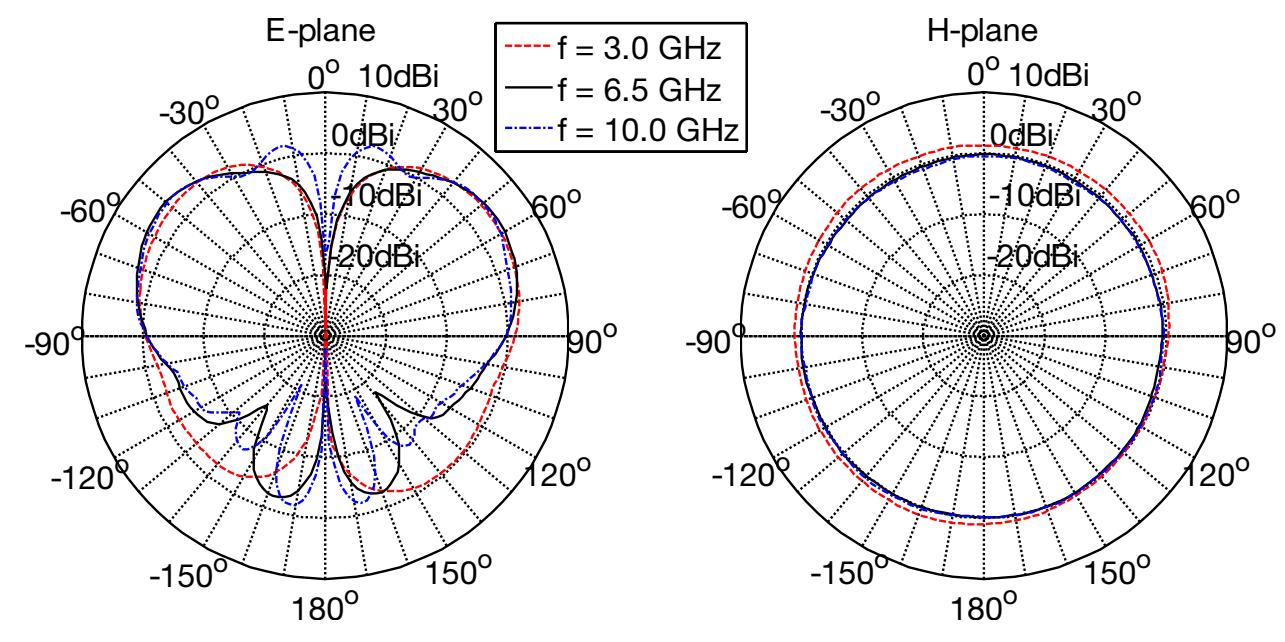

Figure 4: Simulated gain pattern of the conical monopole UWB antennas in E-plane [left] and H-plane [right].

\section{Deconvolution}

\subsection{Ill-posedness}

For a causal time-limited input-output waveform set, $\{x(t), y(t)\}$, where $y(t)=x(t) * h(t)+n(t)$, with $*$ denoting the convolution operator and $n(t)$ being the ambient noise, deconvolution of the channel impulse response (CIR), $h(t)$, is, in general, an ill-posed problem [41]. The ill-posedness in the deconvolution problem has two aspects, first, there is no unique solution, and second, the solution procedure is often unstable. The instability is reflected in the attempt of a direct frequency-domain inversion $H(\omega)=Y(\omega) / X(\omega)$ which leads to erroneous computation of $h(t)$ as the noise component in $Y(\omega)$ may not be small when $X(\omega) \simeq 0$ [42].

The lack of a direct inverse operation gave rise to multiple deconvolution methods. However, only few of them are having super-resolution capability. The non-iterative algorithms are mostly maximum likelihood estimator based, and they suffer heavily from the noise induced instability problems. This makes the iterative CLEAN algorithms a natural choice for deconvolution in noisy environments.

\subsection{Variants of CLEAN}

In this section, we present two versions of the CLEAN algorithm (Algorithm 1 and 2). The basic CLEAN algorithm assumes a linear time-invariant tapped delay line model for the channel, and through successive iterations, extracts the CIR, termed as clean map, by subtracting shifted input signal replicas from the output or dirty map [43]. For the modified CLEAN algorithm, subtraction takes place in the convolution domain [44]. Both the algorithms are readily implementable in popular software packages (e.g. MATLAB) as they work on the discrete version of the time-domain input-output waveforms, $x[n] ; n \in \mathbb{Z}_{\leq N_{1}}^{*}$ and $y[n] ; n \in \mathbb{Z}_{\leq N_{2}}^{*}$. The discrete time index $(n)$ is related to the absolute time $(t)$ through the relation, $t=n / f_{s}$, where $f_{s}$ is the sampling rate mentioned in Section 2.4. Before feeding the sequences, appropriate zero-padding is required to make the length of the sequences equal to $N$, where $N=\max \left\{N_{1}, N_{2}\right\}$. Further, the discrete cross-correlation operator, $\tilde{*}$, is defined as

$$
\mathcal{R}^{x y}[n]=x[n] \tilde{*} y[n]=\hat{R}^{x y}[n-N]
$$



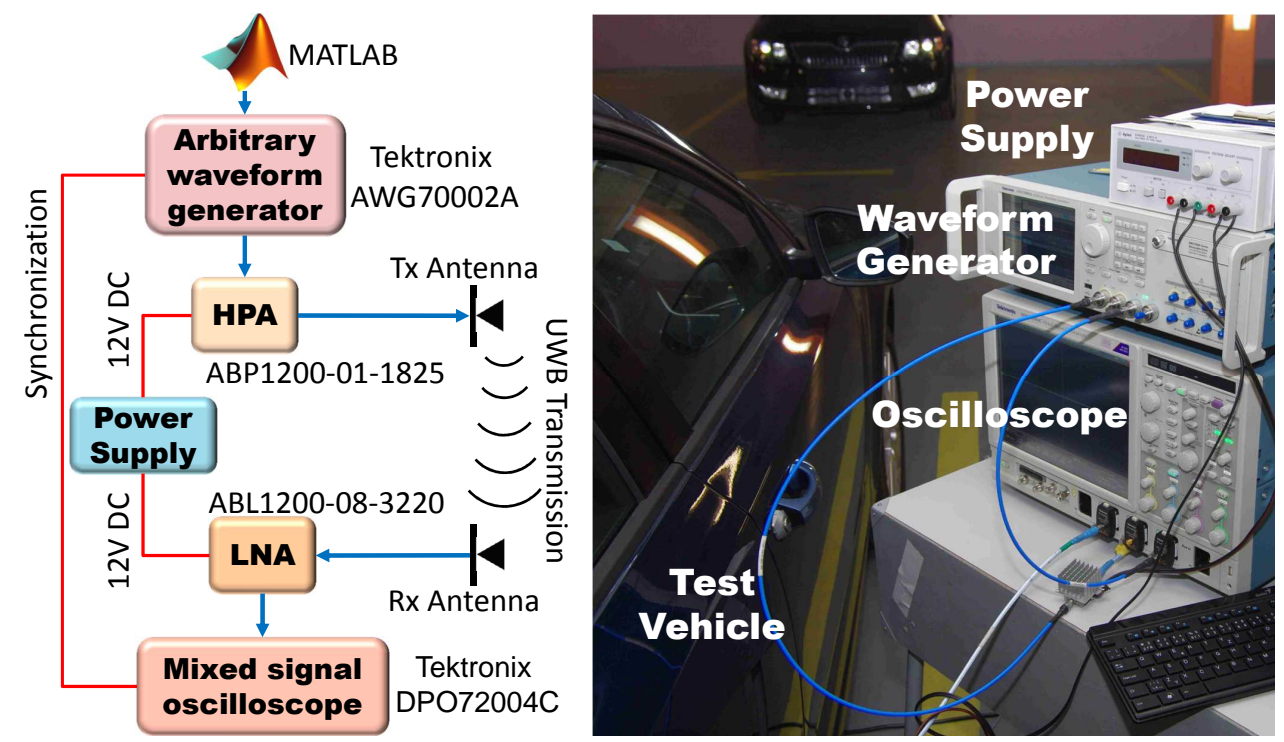

Figure 5: [left] Block diagram of the measurement setup. [right] Photograph of the apparatus assembly.

where

$$
\hat{R}^{x y}[n]= \begin{cases}\sum_{k=0}^{N-n-1} x[n+k] y[k] & \text { for } \quad n \geq 0 \\ \hat{R}^{y x}[-n] & \text { for } \quad n<0\end{cases}
$$

The stopping criterion for successive cleaning may be chosen to attain a target energy capture ratio

$$
E C R=\left[1-\left(\frac{\|y-\hat{y}\|}{\|y\|}\right)^{2}\right] \times 100 \%
$$

where $\hat{y}(t)=x(t) * \hat{h}(t)$ denotes the reconstructed output generated using the estimated response $\hat{h}(t)$, or to maintain a dynamic range

$$
D R=20 \log _{10}\left[\frac{\max _{n}(\hat{h}[n])}{\min _{n}(\hat{h}[n])}\right]
$$

considering only non-zero elements of $\hat{h}$. In this paper, we considered a threshold, $\mathcal{T}$, equal to $10 \%$ of the peak cross-correlation value. A higher value caused missing of significant multi-path components (MPCs), while a lower threshold generated too many paths picking up background noise.

The leverages of the modified algorithm over the basic one are threefold. First, one may notice the presence of loop gain $(\gamma)$ in the basic algorithm (step 7), the choice of which is often empirical and casedependent. Second, the modified algorithm is faster and computationally less intensive as it avoids the calculation of the correlation at each iteration. Third, there is an absolute assignment of a MPC in step 8, which was not possible for the basic CLEAN algorithm. In algorithm 1, a former value of the index $\left(n_{k}^{s}\right)$ might repeat (quite frequently if the choice of $\gamma$ is not optimal) after some iterations, i.e. the residues in the dirty map may result in an update of the clean map. 


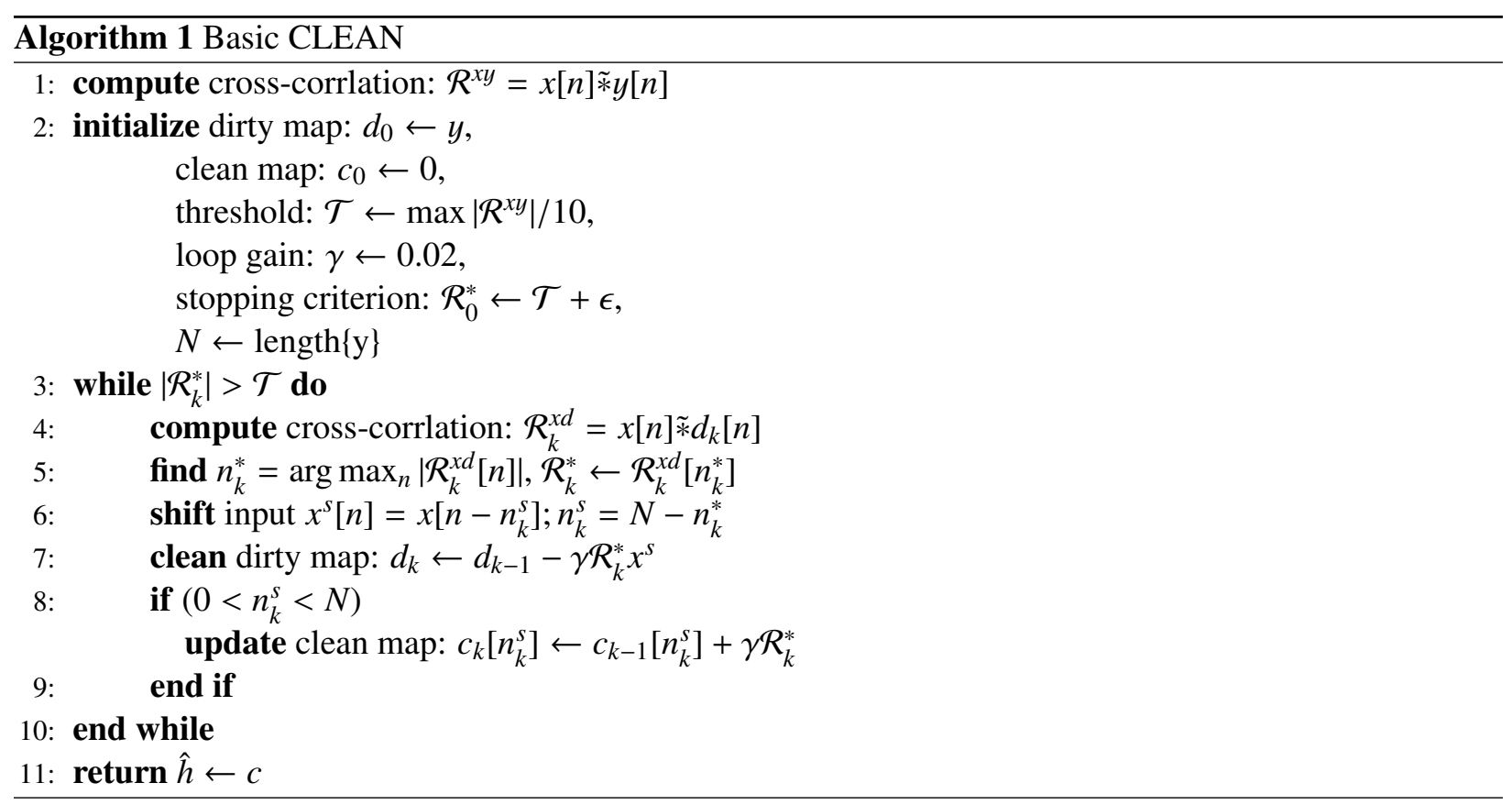

\subsection{Comparison}

For comparison, we have convolved the input signal given in (5) with some energy normalized $\left(\sum_{n} h^{2}[n]=\right.$ 1) synthetic impulse response, added Gaussian noise, and applied the algorithms described in Section 3.2 to estimate the CIRs. Although the common strategy is to match the reconstructed signal $\hat{y}(t)$ against the original received signal $y(t)$, we compared $\hat{h}(t)$ directly with $h(t)$ to see which method provides a better representation of the multipath nature of the channel.
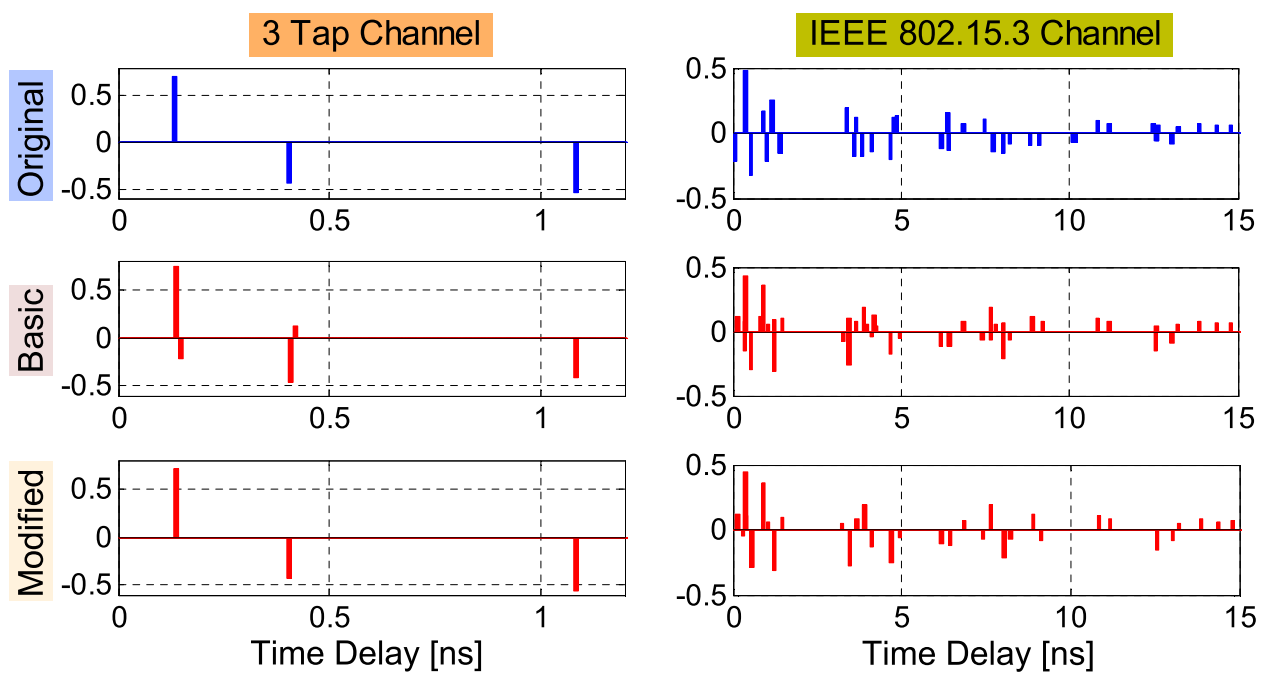

Figure 6: Comparison of the estimated CIRs with the original CIR, SNR $=10 \mathrm{~dB}$.

The test is first performed for a simple 3 tap channel with tap gains separated by a distance less than the pulse width, in order to test the super-resolution potential of CLEAN. A casual inspection of the reconstruc- 


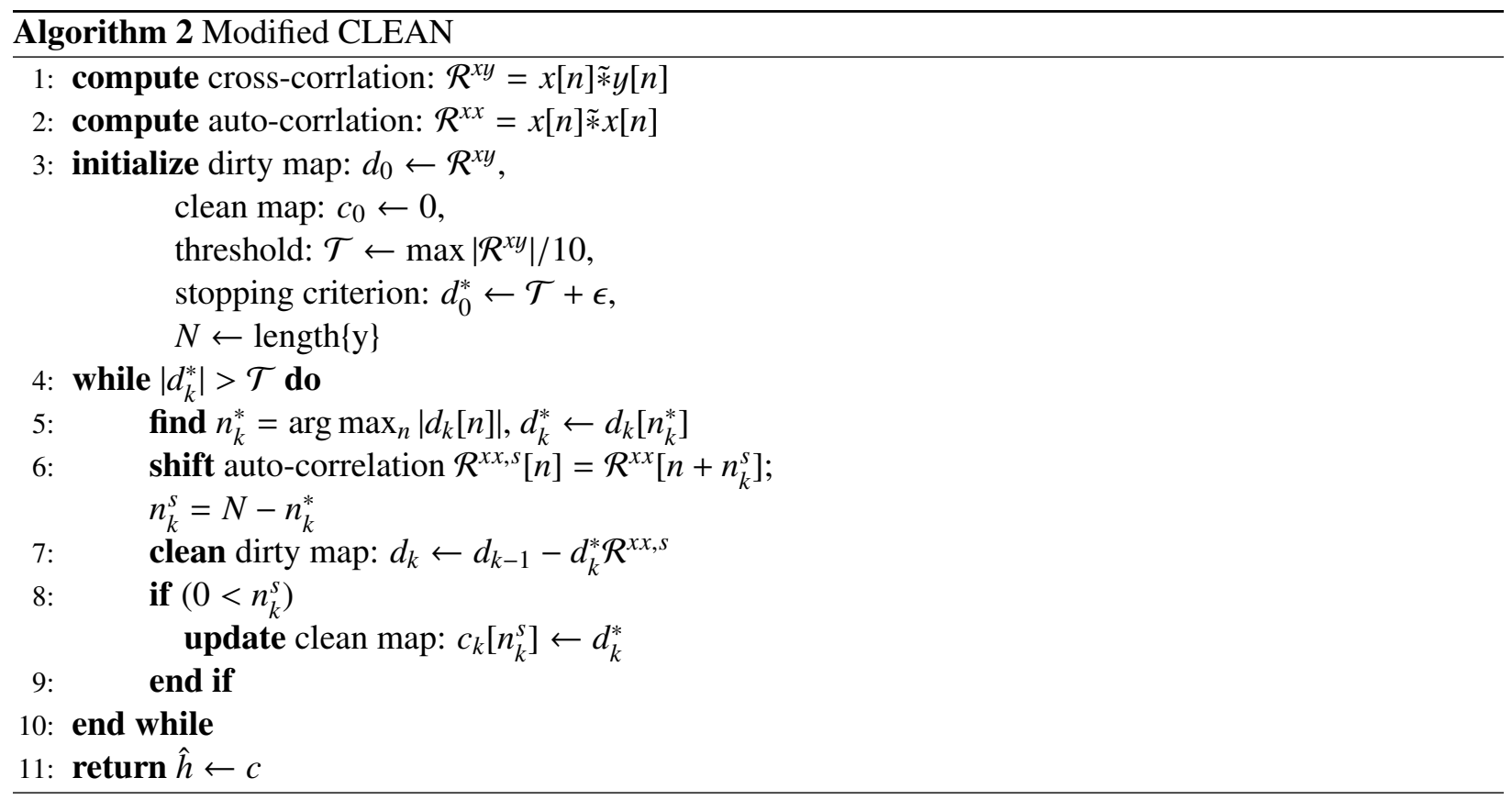

tion results (Fig. 6, left) reveals that the modified method results in less spurious components, and the ratio between multipath tap gains are better maintained.

Next we simulated the discrete version of the standard UWB IEEE 802.15.3 channel based on modified Saleh-Valenzuela model [45]. The model specifies that channel delay taps can be grouped into clusters and the power falls off exponentially from cluster to cluster. Also, within each cluster, the power falls off exponentially from tap to tap. For the sake of simplicity, we have assumed non-overlapping clusters of rays. Further, out of the four specified channel models (CMs), the simulation parameters for only CM1 and CM2 (0-4 m, LoS/ nLoS) were implemented to resemble the intra-vehicular environment, and significant paths within $10 \mathrm{~dB}$ of the peak have been retained.

Comparison of the estimated CIR profiles (Fig. 6, right) is no longer possible through visual inspection. In fact, assessing relative merits of two solutions for an ill-posed problem is subjective to the metric used. As an indicative example, measuring the number of significant MPCs is not very meaningful; the multipath profile depends not only on the number of taps, but also on their respective delays. Another crude method is to compare the mean square error (MSE), $\sum_{n=1}^{N}(\hat{h}[n]-h[n])^{2} / N$. However, the results averaged over 1000 channel samples indicated a marginal improvement (3-4\%) of the MSE when the modified algorithm is used.

A better conclusion may be reached by resorting to a statistical comparisons. We begin with computing the Pearson product-moment correlation coefficient between the CIRs estimated via the two different methods and the original CIR profile

$$
\rho=\frac{\sum_{n=1}^{N}\left(h[n]-\mu_{h}\right)\left(\hat{h}[n]-\mu_{\hat{h}}\right)}{\sqrt{\sum_{n=1}^{N}\left(h[n]-\mu_{h}\right)^{2}} \sqrt{\sum_{n=1}^{N}\left(\hat{h}[n]-\mu_{\hat{h}}\right)^{2}}}
$$

where $\mu_{h}$ and $\mu_{\hat{h}}$ denote the expectations of the original and estimated CIR arrays, respectively, and plot a histogram of their difference. The positive skewness (Fig. 7, left) tells, how often, on average, the CIRs 
constructed with the modified algorithm matches better than that obtained through the basic algorithm. Note that the suffixes, BC and MC, are used to signify the basic CLEAN and modified CLEAN algorithms.
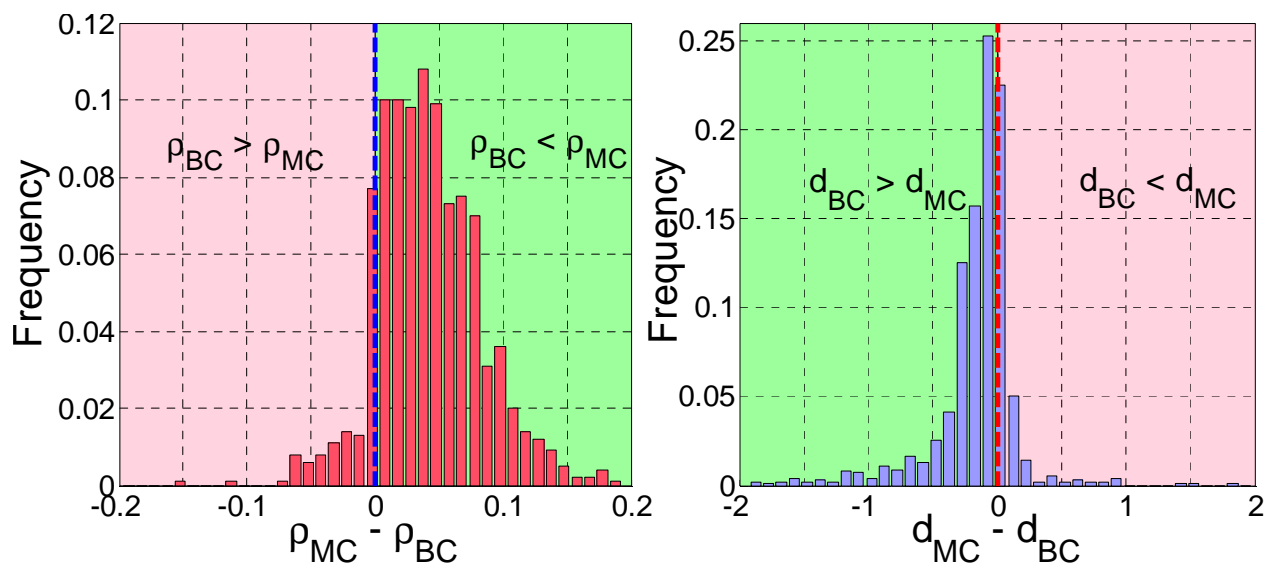

Figure 7: Histogram of difference of correlation coefficient [left] and K-L distance [right].

Another such measure is the Kullback-Leibler (K-L) divergence [46]

$$
d=\sum_{n=1}^{N} h[n] \log _{2}\left(\frac{h[n]}{\hat{h}[n]}\right)
$$

which measures the distance between common non-zero elements of the CIR vector. A negative skewness of the histogram (Fig. 7, right) of the difference of the distances also vouch for the superiority of the modified algorithm.
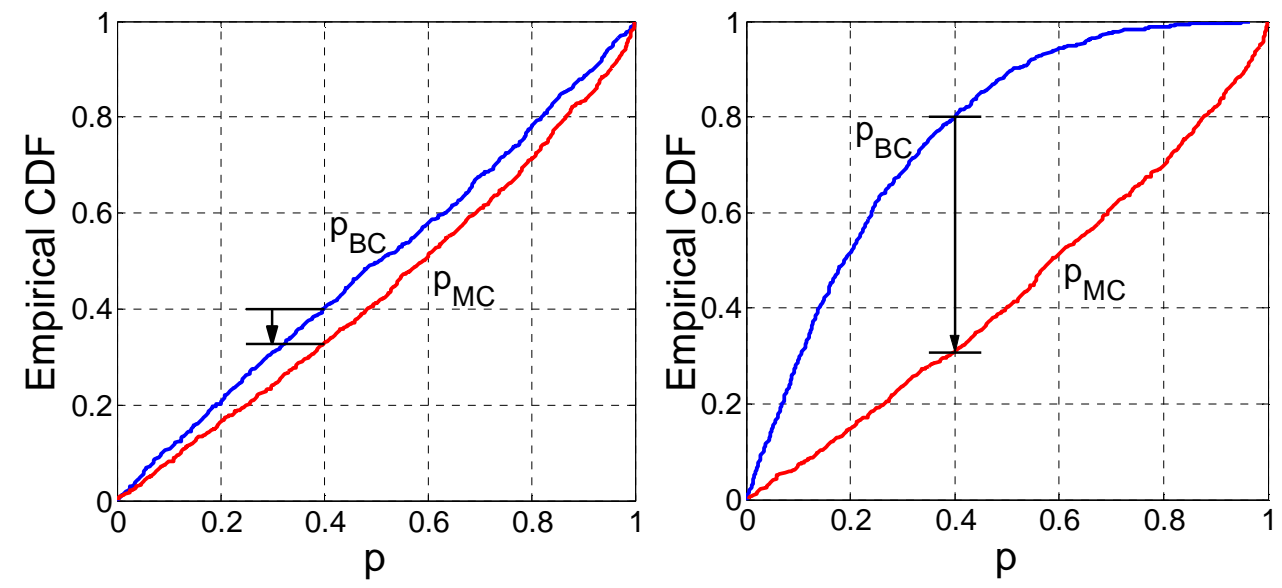

Figure 8: Comparison of CDF $p$-values obtained from K-S test for $\gamma=0.02$ [left] and $\gamma=0.03$ [right].

Finally, a two-sample Kolmogorov-Smirnov (K-S) test is performed. The test is different from the earlier two tests in the sense that it does not judge the one-to-one correlation, rather it focuses on the similarity of the inherent random distribution of CIR tap gains.

The K-S test statistic is defined by [47]

$$
D=\max _{n}\left|F_{\hat{h}}[n]-F_{h}[n]\right|
$$


where $F[n]$ denotes the empirical cumulative distribution function $(\mathrm{CDF})$. The null hypothesis $\left(\mathcal{H}_{0}\right)$ that both $F_{\hat{h}}[n]$ and $F_{h}[n]$ belong to the same family of distribution, is rejected if $D>D_{\alpha}$, where the critical value $D_{\alpha}$ is calculated from the table of Kolmogorov distribution at a given significance level $\alpha$

$$
\operatorname{Pr}\left\{D \leq D_{\alpha}\right\}=1-\alpha
$$

If $F_{D}$ denote the CDF of $D$, the corresponding $p$-value is given by, $p=1-F_{D}\left(D_{\alpha}\right)$.

The Kaplan-Meier estimate of the CDF of the $p$-values, measured against a 5\% significance level $(\alpha=$ $0.05)$, are shown in Fig. 8. Irrespective of the loop-gain parameter $(\gamma)$ value, the CDF for the basic algorithm exceeds the modified one, implying that the probability of obtaining smaller $p$-values for the basic case is higher. The sensitivity of the basic algorithm to parameter setting is also quite self explanatory. When $\gamma$ changes slightly from 0.02 to 0.03 , the gap between the CDFs widens drastically.

\subsection{Post Processing}
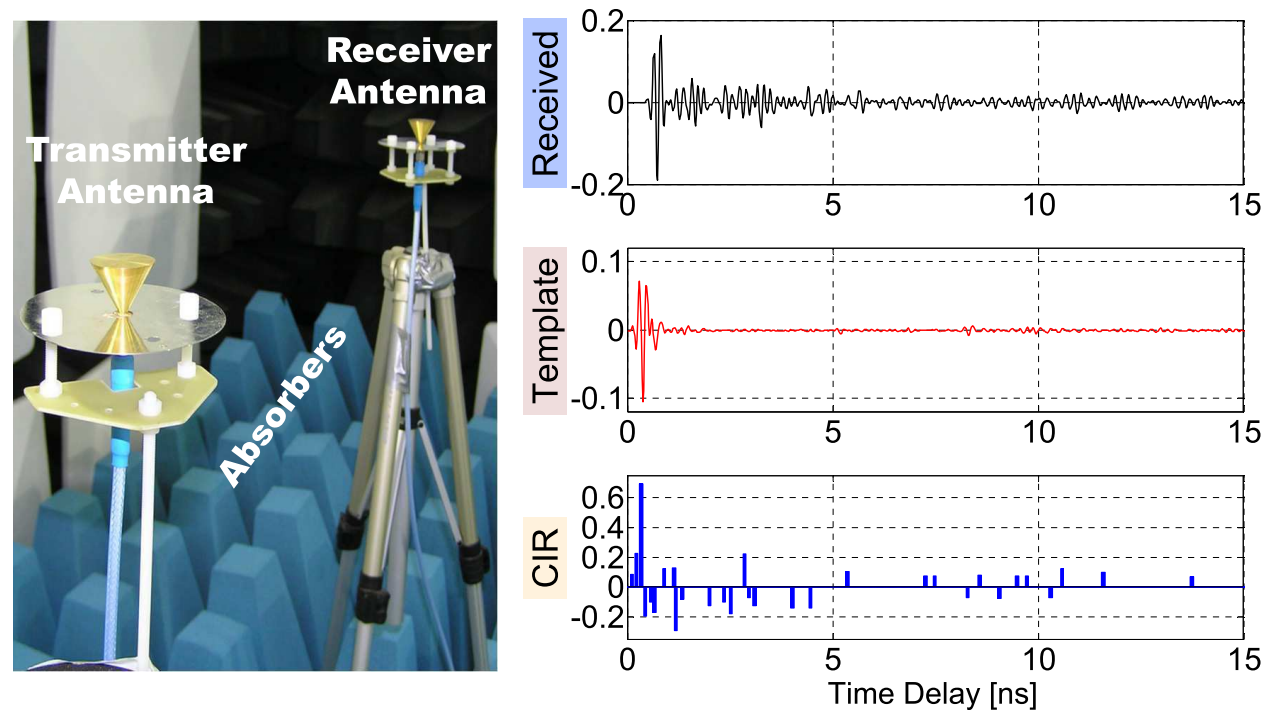

Figure 9: Anechoic chamber configuration [left] and a sample of post-processing of the measured data [right].

For a particular measurement, the received signal, $y(t)$, depends not only on the channel response but also on the measurement system effects, and can be represented as [48]

$$
y(t)=h_{R x, A n t} * h(t) * h_{T x, A n t} * x(t)
$$

where $h_{T x, A n t}$ and $h_{R x, A n t}$ are the impulse responses of the transmitter and receiver antennas along with effects of other attached instruments. For proper calibration, a reference input template

$$
x_{r e f}(t)=h_{R x, A n t} * h_{T x, A n t} * x(t)
$$

was obtained by measuring the response of the input $x(t)$ in an anechoic chamber free from reflections and diffractions (Fig. 9, left). The measurement involved the same set of measurement devices as described in Section 2.4 and the reference distance between Tx and Rx antennas was $1 \mathrm{~m}$. For assessing the need 
of calibration against temporal variations, some measurements were repeated but the variation of delay parameters were found to be negligible.

Next, multipath intensity profiles are obtained by deconvolving the received signals with the input template using the modified CLEAN algorithm. A sample of the post processing of data is shown in Fig. 9 [right]. The entire data set and the post-processing software will be made available at our project documentation webpage [36].

For most of the recorded data sets an energy capture ratio, as denoted in (9), of $60 \%$ to $75 \%$ is obtained. An interesting fact in this connection is that the ECR does not improve when the threshold $(\mathcal{T})$ is lowered, as one would expect. The dynamic range given by (10), however, increses linearly with the threshold. For the parameter values mentioned in Section 3.2, a dynamic range of about $20 \mathrm{~dB}$ is achieved.

\section{Results}

\subsection{Power Delay Profile}

The CIR profiles $(\hat{h}[n])$ obtained after the post-processing via CLEAN can be utilized to construct the power delay profile (PDP) which is a squared-magnitude version of the impulse response. The normalized PDP is calculated from the following relation

$$
P(\tau)=\frac{|\hat{h}[n]|^{2}}{\sum_{n}|\hat{h}[n]|^{2}}
$$

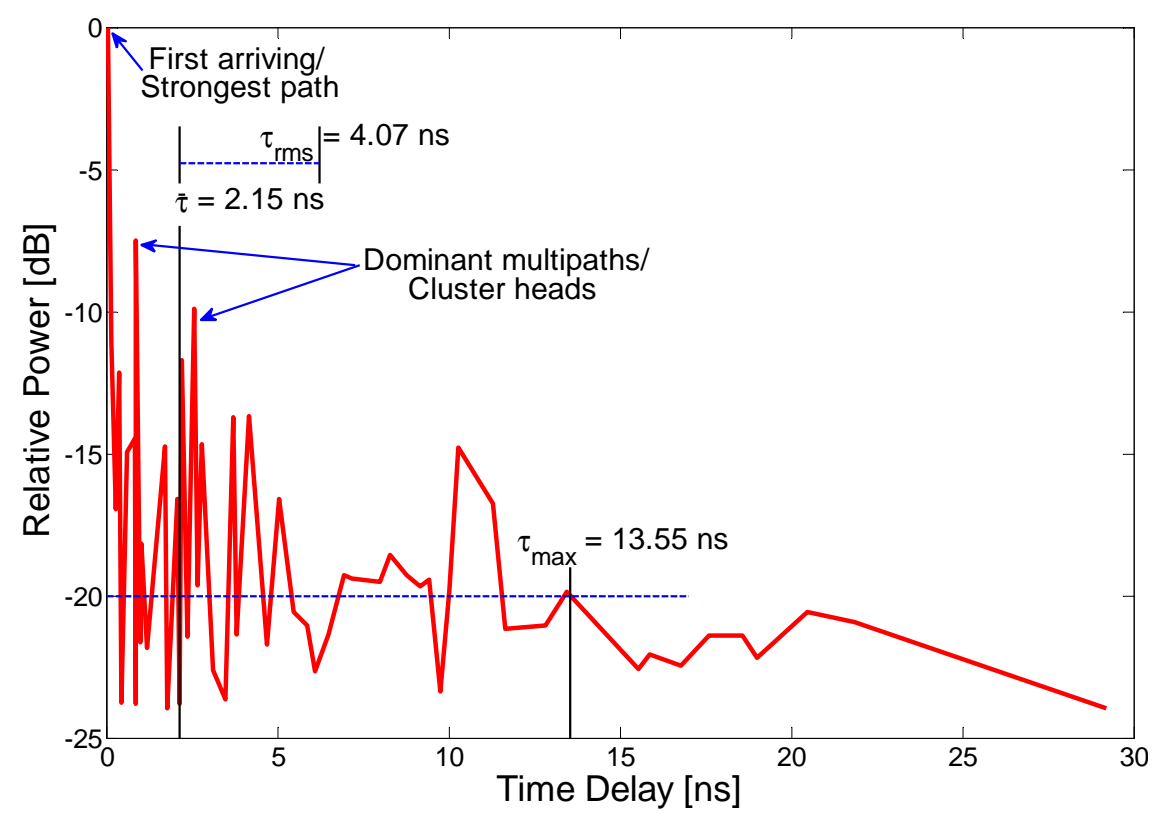

Figure 10: Power delay profile for in-vehicle wireless channel under LoS condition (Tx antenna position: $\mathrm{L}_{2}$, Rx antenna position: $\mathrm{D}$, and Tx-Rx distance is $0.97 \mathrm{~m}$ ).

In Fig. 10, a typical intra car PDP under LoS condition is depicted. The first arriving multipath component is the strongest one which represents the direct LoS transmission. The time scale is adjusted so that the 
this path has zero excess delay $\left(\tau_{0}=0\right)$. The PDP decays fast and has a maximum excess delay of 13.55 ns for a $20 \mathrm{~dB}$ threshold. The mean excess delay and the RMS delay spread (defined in the next subsection) values are also comparatively small.

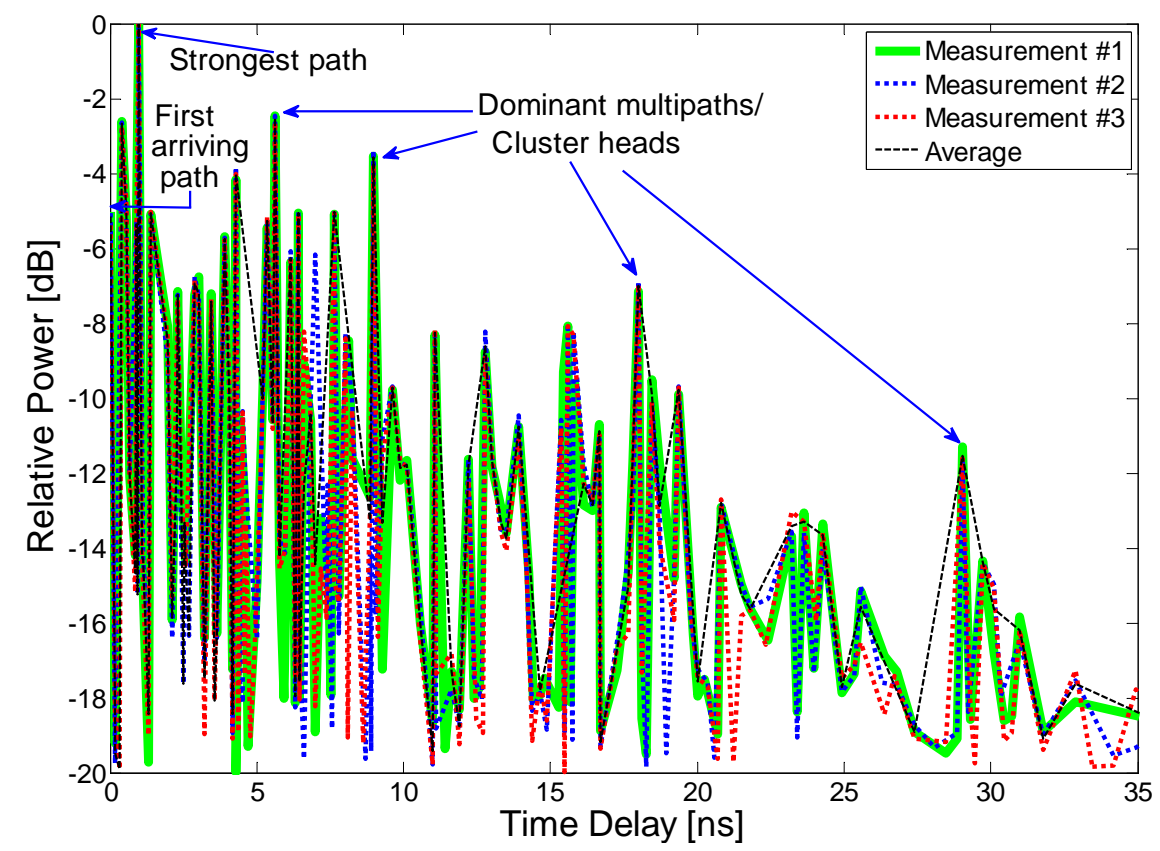

Figure 11: Power delay profile for in-vehicle wireless channel under $\mathrm{nLoS}$ condition (Tx antenna position: $\mathrm{L}_{1}$, Rx antenna position: $\mathrm{P}_{3}$, and Tx-Rx distance is $1.68 \mathrm{~m}$ ).

Fig. 11 shows PDPs when the direct path is obstructed by the seats. We have performed three consecutive measurements keeping the Tx and Rx antenna positions fixed. All the three measurements exhibit peaks at same delay instants. This is somewhat expected because when the car is parked (static condition), the relative positions of reflectors and scatterers do not change much, and the wireless channel in the passenger compartment appears stationary with respect to time. When compared to Fig. 10, one can find that for nLoS condition, the first arriving path is no longer the strongest path and the PDP decays at a lower rate.

From both Fig. 10 and Fig. 11, dominant multipaths can be identified easily. The amplitudes of the dominant paths decay exponentially along the delay axis and multiple other paths exist between two successive dominant peaks. Considering these dominant paths as cluster heads (first path of a multipath cluster) and considering the paths in-between as rays within a cluster, the channel can be characterized following a Saleh-Valenzuela (S-V) model. Different parameters for the S-V model such as cluster amplitude, path amplitude, inter-cluster delay, inter-path delay etc. have been derived extensively in [16] and are not repeated here. The observation is, however, very important as it validates our choice of simulation testbed which is also constructed using a S-V model.

The PDP may be exploited to gain other useful information as well. For example, it is possible to extract a tap-delay model (table of tap gains at different tap delays) by sampling the PDP. Next, utilizing the table, one may simulate the bit error rate (BER) as demonstrated in [49]. It must be noted here that only large scale decaying trend of the PDP is generally considered for such calculations. In another work [50], we have shown that the large scale variation of the PDP for intra-vehicle UWB propagation follows a two-part exponentially decaying function representing the dominant paths and the reverberant diffuse tail. It was also 
demonstrated that the generalized extreme value (GEV) distribution serves as a good fit for modelling the small-scale variations.

\subsection{Time Delay Parameters}

Analysis of PDP is interesting when one compares LoS and nLoS links but we failed to gain much insight from PDPs regarding the effect of passenger occupancy. This lead us to concentrate on the two most common time dispersive quantities that characterize a wideband multipath channel [51], the mean excess delay $(\bar{\tau})$ and the root mean square (RMS) delay spread $\left(\tau_{r m s}\right)$, which are defined as follows

$$
\bar{\tau}=\frac{\sum_{n} \tau_{n}|\hat{h}[n]|^{2}}{\sum_{n}|\hat{h}[n]|^{2}}
$$

and

$$
\tau_{r m s}=\sqrt{\frac{\sum_{n} \tau_{n}^{2}|\hat{h}[n]|^{2}}{\sum_{n}|\hat{h}[n]|^{2}}-\left[\frac{\sum_{n} \tau_{n}|\hat{h}[n]|^{2}}{\sum_{n}|\hat{h}[n]|^{2}}\right]^{2}}=\sqrt{\mathbb{E}\left\{\left(\tau_{n}-\bar{\tau}\right)^{2}\right\}}
$$

where $\tau_{n}$ and $\hat{h}[n]$ are the excess delay and gain associated with the $n$th path of the estimated CIR, respectively, and $\mathbb{E}\{\cdot\}$ is the expectation operator. The mean excess delay and the RMS delay spread can be derived from the first moment and second central moment of the average PDP. Sometimes, the RMS delay spread measure is considered to be more fundamental as it is not much susceptible to apparatus settings and synchronization. Also, it helps in calculating the maximum data rate attainable without inter-symbol interference (ISI). As a rule of thumb, serious ISI is likely to occur at the receiver (without equalizer) when the symbol duration is less than ten times of the RMS delay spread [16].
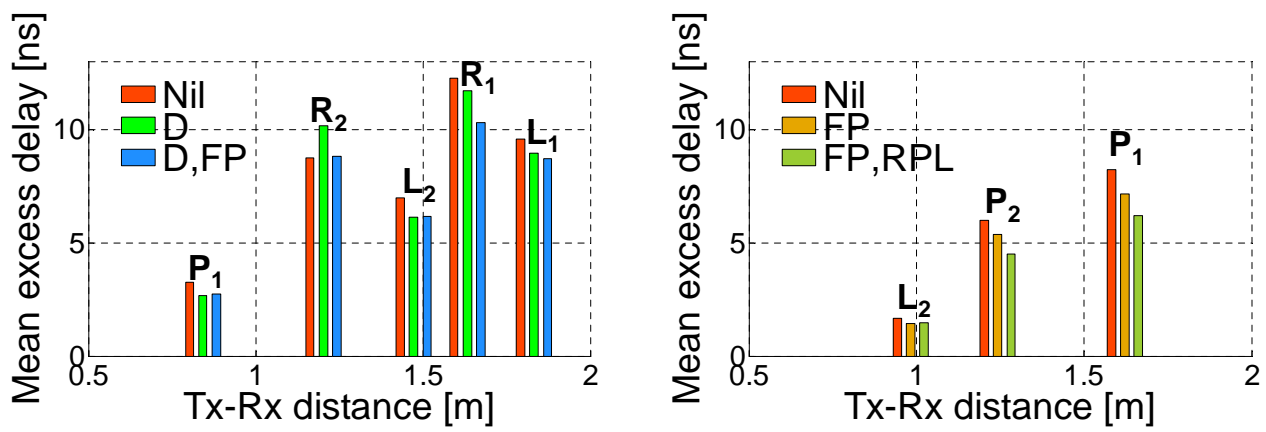

Figure 12: Mean excess delay with varying passenger occupancy when Rx antenna is positioned at RPR [left] and D [right]. Bar group top markings denote Tx antenna position. Passenger occupancy legends - D: driver, FP: front passenger, RPL: rear passenger on left.

Fig. 12 and Fig. 13 plots the mean excess delay and RMS delay spread, respectively, versus the Tx-Rx distance. Both figures display a weak correlation between distance and delay values, and it is difficult to reach to a clear conclusion. Viewed from another angle, it may be said that the delay parameters do not necessarily increase with distance and the most remote wireless user (with respect to the access point) will not necessarily have the worst quality of service. 

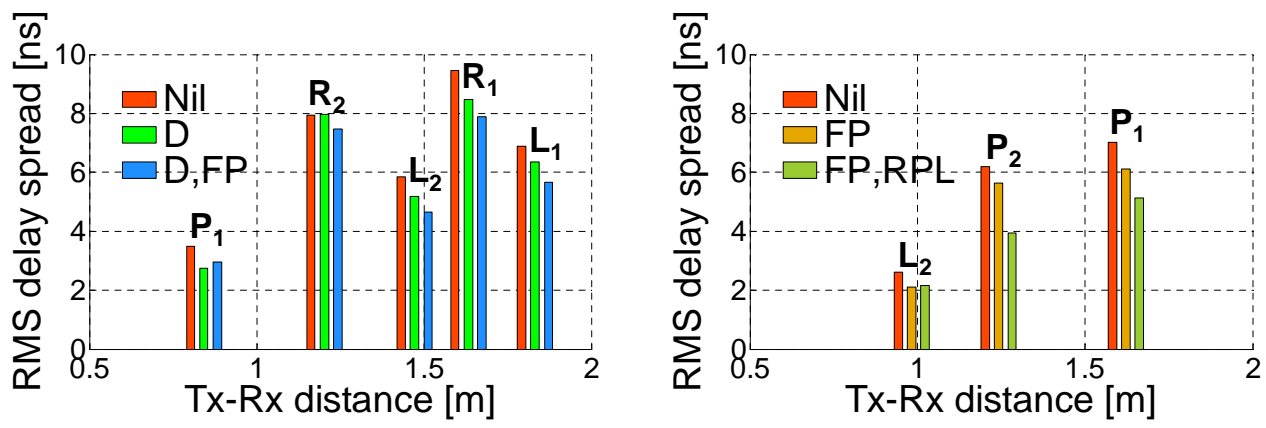

Figure 13: RMS delay spread with varying passenger occupancy when Rx antenna is positioned at RPR [left] and D [right]. Bar group top markings denote Tx antenna position. Passenger occupancy legends - D: driver, FP: front passenger, RPL: rear passenger on left.

More importantly, the figures (Fig. 12 and Fig. 13) also exhibit the effect of passenger occupancy. Considering the bar groups, we find that the decrease in mean delay and delay spread values with increased passenger occupancy is consistent. The reduction reflects that fewer MPCs are available due to obstruction and absorption of several MPCs by the human body. The lower values of $\tau_{r m s}$ with more passengers onboard is very promising for design engineers, because the data rate demand will increase with the number of passengers, and the channel characteristics display that this demand can be met, at least theoretically, by employing adaptive modulation and/or coding.

Finally, we studied how the presence of a direct LoS path affects these two delay parameters. When we compare the data set for the following Tx-Rx pair namely, $\mathrm{L}_{1}-\mathrm{D}$ (LoS, 1.18m) and $\mathrm{R}_{2}-\mathrm{RPR}$ (nLoS, 1.19m), we find that the delay parameters (mean and RMS spread) for the nLoS situations are noticeably larger than that of the LoS scenarios at an equivalent Tx-Rx distance. The reason behind this is the increased number of reflections, as well as signal attenuation, that is incurred in the nLoS case.

\section{Conclusions and Future Work}

In this paper, we compared two versions of the CLEAN algorithm for time domain deconvolution. The efficacy of the modified CLEAN algorithm over the basic version is established through statistical measures. Next, the modified algorithm was used to estimate the CIR in an intra-vehicular UWB channel sounding experiment.

The presence of clusters in the PDPs, obtained after post-processing of channel measurement data, validates the Saleh-Valenzuela assumptions. PDPs also indicated that the passenger compartment wireless channel is time-stationary, when the vehicle is parked.

The time delay parameters obtained from the experimental data were utilized to study the effects of passenger occupancy, Tx-Rx distance, and obstruction of LoS path on intra-vehicle wireless channel parameters. The results show that while the mean excess delay and the RMS delay spread are weakly dependent on the antenna separation, they decrease linearly with passenger occupancy. Further, delay parameter values are considerably smaller when a direct LoS path is present.

The observations in the present text are solely based on the data obtained for the static condition. Currently we are collecting data for a variety of alternate conditions, namely when the engine is turned on, or when the vehicle is moving. It will be interesting to find whether similar trends for delay parameters also exist under all these conditions. Further, a pulse compression based time domain channel sounding system 
has been recently developed at VUT which provides a higher dynamic range due to the inherent correlation gain. We would like to study the suitability of the modified CLEAN algorithm for the new setup.

\section{Acknowledgment}

This work was supported by the SoMoPro II programme, Project No. 3SGA5720 Localization via UWB, co-financed by the People Programme (Marie Curie action) of the Seventh Framework Programme of EU according to the REA Grant Agreement No. 291782 and by the South-Moravian Region. The research is further co-financed by the Czech Science Foundation, Project No. 13-38735S Research into wireless channels for intra-vehicle communication and positioning, and by Czech Ministry of Education in frame of National Sustainability Program under grant LO1401. For research, infrastructure of the SIX Center was used. The generous support from Tektronix, Testovací Technika, and Skoda a.s. Mlada Boleslav are also gratefully acknowledged.

\section{References}

[1] Tuohy S, Glavin M, Hughes C, Jones E, Trivedi M, Kilmartin L. Intra-vehicle networks: A review. IEEE Trans Intell Transport Syst. 2014, DOI: 101109/TITS20142320605 May;p. 1-12.

[2] Held G. Inter- and Intra-Vehicle Communications. Boca Raton, NY, USA: Auerbach; 2008.

[3] Nobuhiro F, Masayoshi M, Atsuo I, Masami G. Intra-and inter-vehicle communication network using low-cost POF links. IEICE Trans Info Syst Tech. 2002 Nov;E85-D(11):1839-50.

[4] Kim JY, Choi HK. An enhanced security protocol for VANET-based entertainment services. IEICE Trans Commun. 2012 Jul;E95-B(7):2245-56.

[5] Ahmed M, Saraydar CU, ElBatt T, Jijun Y, Talty T, Ames M. Intra-vehicular wireless networks. In: Proc. IEEE GLOBECOM. Washington DC, USA; 2007. p. 1-9.

[6] Hatamoto H, Ano S, Kikuchi N, Shimizu S. An evaluation of transmission performance for wireless harness systems using propagation models in an automobile engine compartment. In: Proc. IEEE PIMRC. London, UK; 2013. p. 117-21.

[7] Ohira M, Umaba T, Kitazawa S, Ban H, Ueba M. Experimental characterization of microwave radio propagation in ICT equipment for wireless harness communications. IEEE Trans Ant Prop. 2011 Dec;59(12):4757-65.

[8] Saghir H, Nerguizian C, Laurin JJ, Moupfouma F. In-cabin wideband channel characterization for WAIC systems. IEEE Trans Aerosp Electron Syst. 2014 Jan;50(1):516-29.

[9] Hamada S, Tomiki A, Toda T, Kobayashi T. Wireless connections within spacecrafts to replace wired interface buses. In: Proc. IEEE AERO. Big Sky, Montana, USA; 2013. p. 1-9.

[10] Takahashi K, Udagawa T, Zhang H, Arita T, Nakagawa M. Intra-vehicle wireless 1394 system. IEICE Trans Commun. 2002 May;E85-B(5):938-45. 
[11] Sipal V, Gelabert J, Allen B, Stevens C, Edwards D. Frequency-selective fading of ultrawideband wireless channels in confined environments. IET Microwaves, Antennas \& Propagation. 2011 Aug;5(11):1328-35.

[12] Demir U, Bas CU, Ergen SC. Engine compartment UWB channel model for intravehicular wireless sensor networks. IEEE Trans Veh Tech. 2014 Jul;63(6):2497-2505.

[13] Li B, Zhao C, Zhang H, Sun X, Zhou Z. Characterization on clustered propagations of UWB sensors in vehicle cabin: measurement, modeling and evaluation. IEEE Sensors J. 2013 Apr;13(4):1288-1300.

[14] Bas CU, Ergen SC. Ultra-wideband channel model for intra-vehicular wireless sensor networks beneath the chassis: from statistical model to simulations. IEEE Trans Veh Tech. 2013 Jan;62(1):14-25.

[15] Bellens F, Quitin F, Dricot JM, Horlin F, Benlarbi-Delaï A, Doncker PD. A wideband channel model for intravehicular nomadic systems. Int J Ant Prop. 2011 Jun;2011(468072):1-9.

[16] Niu W, Li J, Talty T. Ultra-wideband channel modeling for intravehicle environment. EURASIP J Wirel Commun Net. 2009 Feb;2009(806209):1-12.

[17] Nakamura R, Kajiwara A. Empirical study on $60 \mathrm{GHz}$ in-vehicle radio channel. In: Proc. IEEE RWS. Santa Clara, CA, USA; 2012. p. 327-30.

[18] Sawada H, Tomatsu T, Ozaki G, Nakase H, Kato S, Sato K, et al. A sixty GHz intra-car multi-media communications system. In: Proc. IEEE VTC. Barcelona, Spain; 2009. p. 1-5.

[19] Blumenstein J, Mikulasek T, Marsalek R, Prokes A, , Zemen T, et al. In-vehicle mm-wave channel model and measurement. In: Proc. IEEE VTC. Vancouver, Canada; 2014. p. 1-6.

[20] Kato A, Sato K, Fujise M, Kawakami S. Propagation characteristics of 60-GHz millimeter waves for ITS inter-vehicle communications. IEICE Trans Commun. 2001 Sep;E84-B(9):2530-39.

[21] Di Benedetto MG, Giancola G. Understanding Ultra Wide Band Radio Fundamentals. New Jersey, NY, USA: Prentice Hall; 2004.

[22] Suzuki Y, Kobayashi T. Ultra wideband signal propagation in desktop environments. IEICE Trans Fundamentals. 2005 Sep;E88-A(9):2272-78.

[23] Dezfooliyan A, Weiner AM. Evaluation of time domain propagation measurements of UWB systems using spread spectrum channel sounding. IEEE Trans Ant Prop. 2012 Oct;60(10):4855-65.

[24] Molisch AF. Wireless Communications. 2nd ed. West Sussex, England: John Wiley \& Sons; 2011.

[25] Candés EJ, Fernandez-Granda C. Towards a mathematical theory of super-resolution. Comm Pure Appl Math. 2014 Jun;67(6):906-56.

[26] Clark BG. An efficient implementation of the algorithm 'CLEAN'. Astron Astrophys. 1980 Sep;89(3):377-78.

[27] Yang W, Zhang N. A new multi-template CLEAN algorithm for UWB channel impulse response characterization. In: Proc. IEEE ICCT. Guilin, China; 2006. p. 1-4. 
[28] Muqaibel A, Safaai-Jazi A, Woerner B, Riad S. UWB channel impulse response characterization using deconvolution techniques. In: Proc. IEEE MWSCAS. vol. 3. Tulsa, OK, USA; 2002. p. 605-08.

[29] Kobayashi T. Measurements and characterization of ultra wideband propagation channels in a passenger-car compartment. IEICE Trans Fundamentals. 2006 Nov;E89-A(11):3089-94.

[30] Kobayashi T. Measurements and characterization of ultra wideband propagation channels in a passenger-car compartment. In: Proc. IEEE ISSSTA. Manaus, Amazon, Brazil; 2006. p. 228-32.

[31] Katayama Y, Tearsaka K, Higashikatsuragi K, Matsunami I, Kajiwara A. Experimental evaluation of in-vehicle UWB radio propagation characteristics. IEICE Trans Fundamentals. 2006 Sep;J89$\mathrm{B}(9): 1815-19$.

[32] Chandra A, Blumenstein J, Mikulasek T, Vychodil J, Pospisil M, Marsalek R, et al. CLEAN algorithms for intra-vehicular time-domain UWB channel sounding. In: Proc. PECCS. Angers, France; 2015. p. 224-29.

[33] Santos T, Karedal J, Almers P, Tufvesson F, Molisch AF. Scatterer detection by successive cancellation for UWB - method and experimental verification. In: Proc. IEEE VTC. Singapore; 2008. p. 445-49.

[34] Feder M, Weinstein E. Parameter estimation of superimposed signals using the EM algorithm. IEEE Trans Acoust, Speech, Signal Processing. 1988 Apr;36(4):477-89.

[35] Fleury BH, Tschudin M, Heddergott R, Dahlhaus D, Pedersen KI. Channel parameter estimation in mobile radio environments using the SAGE algorithm. IEEE J Sel Areas Commun. 1999 Mar;17(3):434-50.

[36] Department of Radio Electronics, Faculty of Electrical Engineering and Communication, Brno University of Technology. Research into wireless channels for intra-vehicle communication and positioning, GACR-13-38735S: Project documentation page;. URL: http://www.radio.feec.vutbr.cz/GACR13-38735S/.

[37] Federal Communications Commission. Revision of Part 15 of the Commission's Rules Regarding Ultra-Wideband Transmission Systems: First report and order; 2002. FCC 02-48. URL: www.fcc.gov/Bureaus/Engineering_Technology/Orders /2002/fcc02048.pdf.

[38] Ghavami M, Michael LB, Kohno R. Ultra Wideband Signals and Systems in Communication Engineering. 2nd ed. West Sussex, England: John Wiley \& Sons; 2007.

[39] Blumenstein J, Mikulasek T, Marsalek R, Prokes A, Mecklenbrauker C. Measurements of ultra wide band in-vehicle channel - statistical description and TOA positioning feasibility study. In: TWG-V Meeting. Ferrara, Italy; 2014. p. 1-8.

[40] Taniguchi T, Maeda A, Kobayashi T. Development of an omnidirectional and low-VSWR ultra wideband antenna. Int J Wireless Opt Commun. 2006 Aug;3(2):145-57.

[41] Sarkar TK, Tseng FI, Rao SM, Dianat SA, Hollmann BZ. Deconvolution of impulse response from time-limited input and output: Theory and experiment. IEEE Trans Instrum Meas. 1985 Dec;IM34(4):541-46. 
[42] Yang L. The Applicability of the Tap-Delay Line Channel Model to Ultra Wideband [MS Thesis]. Virginia Polytechnic Institute and State University. Blacksburg, VA, USA; 2004.

[43] Vaughan RG, Scott NL. Super-resolution of pulsed multipath channels for delay spread characterization. IEEE Trans Commun. 1999 Mar;47(3):343-47.

[44] Liu TCK, Kim DI, Vaughan RG. A high-resolution, multi-template deconvolution algorithm for timedomain UWB channel characterization. Can J Elect Comput Eng. 2007 Dec;32(4):207-13.

[45] Molisch AF, Jeffrey RF, Marcus P. Channel models for ultrawideband personal area networks. IEEE Wireless Commun. 2003 Dec;10(6):14-21.

[46] Kulhandjian H, Melodia T. Modeling underwater acoustic channels in short-range shallow water environments. In: ACM WUWNet. Rome, Italy; 2014. p. 1-5.

[47] Simard R, L'Ecuyer P. Computing the two-sided Kolmogorov-Smirnov distribution. J Stat Softw. 2011 Mar;39(11):1-18.

[48] Liu L, Wang Y, Zhang Y. Ultrawideband channel measurement and modeling for the future intravehicle communications. Microw Opt Tech Lett. 2012 Feb;54(2):322-26.

[49] Kukolev P, Chandra A, Mikulasek T, Prokes A, Zemen T, Mecklenbrauker C. In-vehicle channel sounding in the 5.8 GHz band. EURASIP J Wirel Commun Net. 2015 Mar;2015(57):1-9.

[50] Blumenstein J, Mikulasek T, Marsalek R, Chandra A, Prokes A, Zemen T, et al. In-vehicle UWB channel measurement, model and spatial stationarity. In: IEEE VNC. Paderborn, Germany; 2014. p. 77-80.

[51] Maas D, Firooz MH, Zhang J, Patwari N, Kasera SK. Channel sounding for the masses: Low complexity GNU 802.11b channel impulse response estimation. IEEE Trans Wireless Commun. 2012 Jan;11(1):1-8. 